\title{
EDITORIAL
}

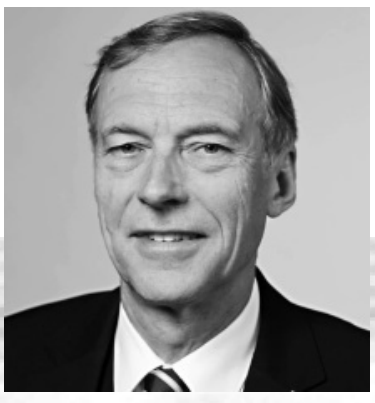

Arndt Bode

Hauptherausgeber

\section{Informatik und Informatiker/innen}

Medizin ohne Informatik, das ist heute kaum denkbar, aber profitiert auch umgekehrt die Informatik von der Medizin bzw. der Medizinischen Informatik? Diesen Perspektivenwechsel vollziehen Klaus Pommerening, Mainz, Thomas M. Deserno, Aachen, Josef Ingenerf, Lübeck, Richard Lenz, Erlangen und Paul Schmücker, Mannheim, die zunächst eine breite Übersicht über das Anwendungsfach Medizinische Informatik geben und dann zu dem Schluss kommen, dass für die Bildverarbeitung, Informationssysteme, Prozessunterstützung, Klassifikation und Terminologien bzw. Ontologien sowie datenschutzfördernde Techniken und Beweis- und IT-Sicherheit die Kerninformatik durch Arbeiten in der Medizinischen Informatik bereichert wird.

„Big Data, Big Opportunities" ist zugleich Titel und Untersuchungsgegenstand des Beitrags von Stefan Wrobel, Hans Voss, Joachim Köhler, Uwe Beyer und Sören Auer, alle am Fraunhofer Institut für Intelligente Analyse und Informationssysteme der Fraunhofer Gesellschaft in St. Augustin sowie an der Universität Bonn. Nach einer Definition des breiten Arbeitsgebiets von Big Data haben die Autoren mehrere Umfragen zur Nutzung dieser Technik in Unternehmen weltweit analysiert. Sie kommen zu dem Schluss, dass Big Data definitiv in der Wirtschaft angekommen ist, dass wir aber, vor allem in Deutschland, Forschungsbedarf zu Big Data haben und vermehrt Ausbildungskapazitäten schaffen müssen.

Ute Schmid, Anja Gärtig-Daugs und Silvia Förtsch von der Universität Bamberg haben sich aufgrund profunder Analysen mit der Frage beschäftigt, warum Frauen in der Informatik nach wie vor eine rare Spezies darstellen. „Introvertierte Studenten, fleißige Studentinnen" fanden sie als geschlechtsspezifische Unterschiede in Motivation, Zufriedenheit und Wahrnehmungsmustern bei Informatikstudierenden. Sie ziehen dabei nicht nur Schlüsse aus umfangreichen Befragungen einer großen Zahl von Studierenden an der Universität Bamberg, sondern analysieren auch Erkenntnisse weltweiter Studien zu diesem Thema. Die Antworten im Artikel sind differenziert und sollten uns allen zu denken geben.

Im Aktuellen Schlagwort wird das neue Entwurfsparadigma Approximate Computing von Christian Plessl, Marco Platzner und Peter J. Schreier, Informatiker und Elektrotechniker der Universität Paderborn präsentiert. Der Grundgedanke entspricht einem althergebrachten Entwurfsprinzip der (Rechner-)Architektur: der Sparsamkeit. Anders ausgedrückt: Wieviel Chipfläche oder Energie bei der Berechnung oder Berechnungszeit kann ich sparen, wenn ich geringfügig auf Genauigkeit verzichte, also approximiere. Die Autoren zeigen, dass es dafür ganz unterschiedliche Realisierungsalternativen von automatischem Lernen bis zum Schaltwerksentwurf gibt, die im Approximate Computing untersucht werden.

Korrelation und Visualisierung von Twitter Hashtags hat Ricardo Usbeck in seiner Masterarbeit an der Universität Leipzig untersucht. Seine Ergebnisse sind im neuesten Beitrag der Student's Corner zusammengefasst.

Drei Beiträge der Rubrik „Zur Diskussion gestellt" sollen uns zum Nachdenken anregen: Heinz-Jürgen Burkhardt, Darmstadt und Rainer Prinoth, Langen, beklagen den Zustand der heutigen IT und des Internets. Nane Kratzke, Lübeck, ärgert sich über überflüssige oder zumindest übertriebene Sperrvermerke bei studentischen Abschlussarbeiten mit Partnern in der Wirtschaft und Thomas Hoppe, Berlin, präsentiert seine Ideen zum Prinzip der Unwahrnehmbarkeit.

Das alles, aber auch noch viel mehr, z. B. Dueck-beta-inside, Forum und GI-Vorstandsperspektive finden Sie in dieser Ausgabe des Informatik-Spektrums. Ich wünsche erbauliche und anregende Lektüre.

\section{Arndt Bode}

DOI 10.1007/s00287-015-0914-9 